\title{
Optimization of relative wear ratio during EDM of titanium alloy using advanced techniques
}

\author{
Neeraj Agarwal ${ }^{1}$ (D) Nitin Shrivastava ${ }^{1} \cdot$ M. K. Pradhan $^{2}$
}

Received: 13 August 2019 / Accepted: 9 December 2019 / Published online: 17 December 2019

(c) Springer Nature Switzerland AG 2019

\begin{abstract}
This study presents the relative wear ratio (RWR) optimization of Titanium alloy 685 for electric discharge machining (EDM). Titanium alloys are widely used in modern industry due to its excellent engineering property like high strength to weight ratio, corrosion resistance. There is difficulty in the machining of titanium alloy with conventional machining processes, hence EDM used to machine titanium alloy. The objective is to maximize the material removal rate (MRR) while the tool wear rate (TWR) should be minimized. The machined surface is an exact replica of the tool face. If TWR increases then the quality of the machined surface deteriorate. A new term introduces a RWR which represents the ratio of MRR to the TWR. The high value of RWR is desired to improve the quality of the machined surface. In this paper RWR is optimized. Response surface model used to develop a regression model of RWR. Optimization of RWR is done by advanced optimization techniques, Jaya Algorithm, teaching-learning-based optimization and response optimizer. Parameters optimization of peak current (Ip), pulse on time (Ton), duty factor (t) and voltage (V) done in this study to maximize RWR.
\end{abstract}

Keywords EDM - Electric discharge machining · Modelling · Optimization · Advanced optimization · Relative wear ratio (RWR) · Titanium alloy $685 \cdot$ Jaya Algorithm

\section{Introduction}

Nowadays there are requirements of advanced materials like superalloys, Titanium alloy, ceramic, etc with excellent engineering property like hardness, strength, high strength to weight ratio, etc. Due to the hardness of these materials, machining is extremely difficult and even sometimes not possible to machine, with conventional machining processes. These materials can be machined by unconventional machining processes like Electrical Discharge Machining (EDM), Electro-Chemical Machining (ECM), Laser Beam machining (LBM), etc [1, 2]. EDM is most popular because of its machining capabilities and comparatively low cost. EDM is a nonconventional machining process, which is used to machine extremely hard material [3-5]. There are two electrodes, one is a workpiece and the other is the tool electrode. Two electrodes are completely submerged in a dielectric fluid. A gap is maintained between two electrodes. Voltage is gradually applied between two electrodes. Due to this potential difference, there is a series of sparking takes place between two electrodes (one is a workpiece and the other is known as a tool/electrode). Due to this sparking, some material from the surface of the workpiece is melt and flushed away from the machining area [6] and some material from the tool surface also removed [7]. There are many control parameters of electrical discharge machining (EDM). Peak current (Ip) is the maximum current flow between electrodes, pulse on time (Ton) is the time interval during each spark, duty factor ( $t$ ) is the percentage of the ratio between pulse duration and total cycle time, voltage (V) is applied a potential difference between both electrodes [8].

Neeraj Agarwal, neeraj.bhopal@gmail.com | 'University Institute of Technology, Rajiv Gandhi Proudyogiki Vishwavidyalaya, Bhopal, India. ${ }^{2}$ Maulana Azad National Institute of Technology, Bhopal, India. 
Higher material removal rate (MRR) is desirable, but higher MRR also leads to a higher tool wear rate (TWR). High TWR deforms the tool shape, which affects the machining quality of the workpiece. Because the machined surface is an exact replica of the tool surface. This limitation can be overcome by maximizing the relative wear ratio (RWR). RWR is the ratio of MRR to TWR (in other words RWR $=$ MRR/TWR). There is a challenge to maximize RWR. Qudeiri Jaber E. Abu et al. reviewed the EDM machinability of Titanium alloy [9]. Relative wear ratio is a very important term for electrical discharge machining (EDM). A higher value of RWR ensures a better quality of EDM machining. To understand the behavior of the process, it is required to develop a model between the process parameters and response measure. Muthuramalingam and Mohan reviewed on the influence of various electrical process parameters in the EDM process [10]. Ghoreishi and Atkinson used RSM to develop a regression model for MRR, TWR, and SR during UAEDM of tool and die steel [11]. Pradhan and Biswas used RSM to development of the model and work on multi-objective optimization of MRR and surface roughness [12]. EDM has many control parameters like peak current, discharge voltage, pulse on-time, pulseoff time, electrode polarity, electrode gap, dielectric [13]. Four important control parameter Peak current (Ip), discharge voltage $(\mathrm{V})$, pulse on time (Ton) and duty factor ( $\mathrm{t}$ ) is selected as control parameter; RWR is selected as quality measures. To prepare the empirical model, thirty experiments were conducted using a design of experiment with central composite design (CCD) [14]. Once the model is prepared, the developed model is used for optimization.

Optimization is the process of finding the best result within the given resources. Rajesh and Dev optimize MRR and surface roughness (Ra) on the EDM process [15]. Kao et al. optimized electrode wear ratio (EWR), MRR and Ra [16]. Tarng et al. find the optimum level of control parameters in wire electrical discharge machining [17]. Many researchers applied different optimization techniques like Genetic Algorithm (GA), simulated annealing (SA), particle swarm analysis (PSA) and many more. These optimization techniques have algorithm-specific parameters and complicated in nature. Rao invented an optimization algorithm, known as Jaya algorithm in 2016. Jaya algorithm is an advanced optimization technique and simpler to implement, further, it requires fewer parameters. Jaya Algorithm is successfully used in constrained optimization as well [18-20]. Teaching-learning- based optimization (TLBO) is an advanced optimization technique that is based on the process of teaching and learning of a student [21,22].

In this paper, RWR has been optimized, through advanced optimization technique Teaching learning based optimization (TLBO) and Jaya Algorithm. RWR optimization of Titanium alloy 685 is discussed in this research

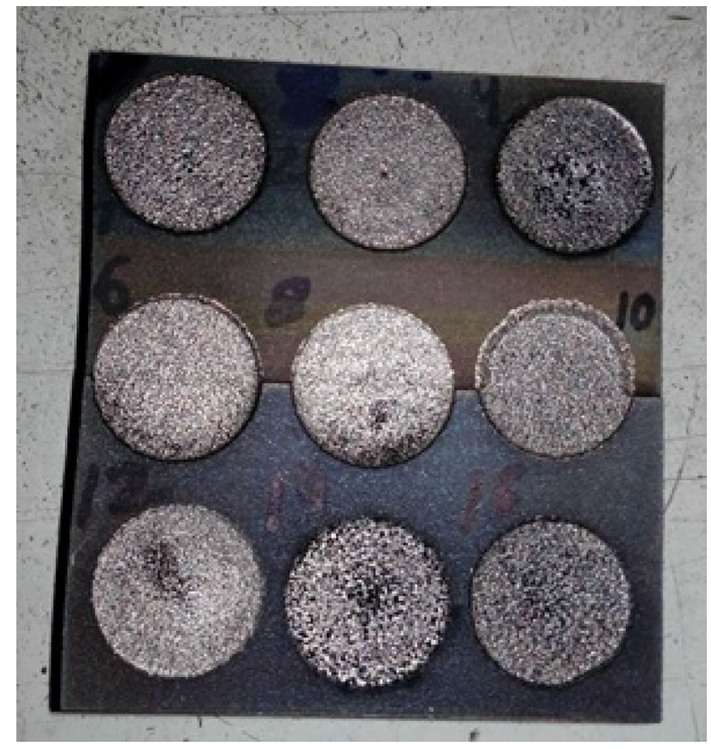

Fig. 1 Workpiece

Table 1 Control parameters and their ranges

\begin{tabular}{lclr}
\hline Parameter & Level 1 & Level 2 & Level 3 \\
\hline Ip (Ampere) & 4 & 6 & 8 \\
Ton ( $\mu$ seconds) & 50 & 100 & 150 \\
Duty Factor (\%) & 25 & 37.5 & 50 \\
V (Volt) & 40 & 70 & 100 \\
\hline
\end{tabular}

paper. No work has been reported on EDM machinability of Titanium alloy 685. Many researchers worked on the optimization of MRR and surface roughness but very few research work reported on RWR optimization. The researcher involves maximizing MRR only (this also maximizes TWR with poor machining quality especially for complicated tool shape). This paper maximizes MRR while TWR is minimized simultaneously.

\section{Experimental setup}

Titanium alloy 685 is selected as a workpiece and a copper rod of $10 \mathrm{~mm}$ diameter is selected as an electrode. Thirty experiments were conducted as per the design of experiments (DOE) shown in Table 2. Four important parameter Ip, Ton, $t$, and $V$ are selected as control parameters. Table 1 shows the corresponding range of variables. Each experiment was conducted for thirty minutes. The workpiece is shown in Fig. 1. The material removal rate (MRR) and tool wear rate (TWR) is recorded for each experiment. The MRR is calculated as following 
Table 2 Experimental details

\begin{tabular}{|c|c|c|c|c|c|}
\hline Exp. & Ip (Amp) & Ton $(\mu \mathrm{s})$ & $t(\%)$ & $\mathrm{V}(\mathrm{V})$ & RWR \\
\hline 1 & 4 & 50 & 25 & 40 & 7.4365 \\
\hline 2 & 4 & 50 & 50 & 40 & 7.9299 \\
\hline 3 & 4 & 150 & 25 & 40 & 21.4429 \\
\hline 4 & 4 & 150 & 50 & 40 & 34.4082 \\
\hline 5 & 4 & 100 & 37.5 & 70 & 31.2013 \\
\hline 6 & 4 & 50 & 25 & 100 & 6.8743 \\
\hline 7 & 4 & 50 & 50 & 100 & 9.5447 \\
\hline 8 & 4 & 150 & 25 & 100 & 15.7667 \\
\hline 9 & 4 & 150 & 50 & 100 & 28.2391 \\
\hline 10 & 6 & 100 & 37.5 & 40 & 9.8320 \\
\hline 11 & 6 & 50 & 37.5 & 70 & 5.2024 \\
\hline 12 & 6 & 100 & 25 & 70 & 8.6039 \\
\hline 13 & 6 & 100 & 37.5 & 70 & 10.0938 \\
\hline 14 & 6 & 100 & 37.5 & 70 & 9.2377 \\
\hline 15 & 6 & 100 & 37.5 & 70 & 8.8455 \\
\hline 16 & 6 & 100 & 37.5 & 70 & 10.4862 \\
\hline 17 & 6 & 100 & 37.5 & 70 & 9.2038 \\
\hline 18 & 6 & 100 & 37.5 & 70 & 9.6536 \\
\hline 19 & 6 & 100 & 50 & 70 & 11.8069 \\
\hline 20 & 6 & 150 & 37.5 & 70 & 15.2702 \\
\hline 21 & 6 & 100 & 37.5 & 100 & 9.6188 \\
\hline 22 & 8 & 50 & 25 & 40 & 3.2859 \\
\hline 23 & 8 & 50 & 50 & 40 & 4.3258 \\
\hline 24 & 8 & 150 & 25 & 40 & 8.1091 \\
\hline 25 & 8 & 150 & 50 & 40 & 13.1414 \\
\hline 26 & 8 & 100 & 37.5 & 70 & 6.5890 \\
\hline 27 & 8 & 50 & 25 & 100 & 3.2556 \\
\hline 28 & 8 & 50 & 50 & 100 & 9.3455 \\
\hline 29 & 8 & 150 & 25 & 100 & 9.8446 \\
\hline 30 & 8 & 150 & 50 & 100 & 13.4870 \\
\hline
\end{tabular}

$M R R=\frac{M_{f w}-M_{i w}}{T_{p}}$

where $M_{f w}=$ Final weight of workpiece after machining, $M_{i w}=$ Intial weight of workpiece, $T_{p}=$ Time period of machining in minutes.

The TWR is calculated as following

$T W R=\frac{E_{f w}-E_{i w}}{T_{p}}$

where $E_{f w}=$ Final weight of tool electrode after machining, $E_{i w}=$ Intial weight of electrode, $T_{p}=$ Time period of machining in minutes.

Relative wear ratio (RWR) is the ratio of MRR to TWR. RWR is calculated for each experiment and inserted into Table 2 to show the experimental result.

RWR is calculated as follwing
$R W R=\frac{M R R}{T W R}$

\section{Modelling and optimization}

\subsection{Modelling}

In response surface methodology, The relation between the quality measures and process control parameters is express as following:

$y=f\left(x_{1}, x_{2}, x_{3}, x_{4}, x_{5}, \ldots x_{n}\right)$

where $x_{1}, x_{2}, x_{3}, x_{4}, x_{5}, \ldots, x_{n}$ are input parameters and $y$ is output response. With plotting the response ' $y$ ', a surface is obtained which is known as response surface. Function of ' $y$ ' may be very complicated and unknown. The RSM aims is to approximating $\mathrm{f}$ by a suitable lower order equation. $A$ second order regression model is represented as following:

$y=b_{0}+\sum_{i=1}^{p} b_{i} x_{i}+\sum_{i=1}^{p} b_{i i} x_{i}^{2}+\sum_{i} \sum_{j} b_{i j} x_{i} x_{j}$

where $b_{0}$ is constant and all $\mathrm{b}^{\prime}$ are regression coefficient.

Response surface methodology (RSM) used to develop a regression model. Commercial statistical software Minitab 18 is used to develop a regression model as following.

$$
\begin{aligned}
R W R= & 39.4 * 21.62 * I p+0.669 * V+0.235 * \text { Ton } \\
& -0.027 * t+1.699 * I p * I p-0.00516 * V * V \\
& +0.0186 * I p * V-0.0273 * I p * \text { Ton } \\
& -0.000659 * V * \text { Ton }+0.00238 * \text { Ton } * t
\end{aligned}
$$

\subsection{Optimization using Jaya Algorithm}

Jaya algorithm is an advanced optimization technique and found suitable for engineering optimization. Figure 2 shows flow diagram of Jaya Algorithm.

Step 1: Initial population is generated randomly with in the range as per Table 1

Step 2: The objective is to maximize RWR as per equation 6. The highest value of RWR, considered as best and lowest RWR considered as worst. Candidate 4 having best value of RWR and corresponding variables of candidate 4 have Ip_best $=4.9262$, Ton_best $=142.7235$, t_best $=47.38632$, V_best $=71.1816$. Candidate 5 having minimum RWR, hence lp_worst $=4.8570$, Ton_worst $=67.9034, \mathrm{t} \_$worst $=39.2846, \mathrm{~V} \_$worst $=51.5042$. 
Fig. 2 Flow diagram of Jaya Algorithm
Initialize population size, Number of design variables, termination criteria

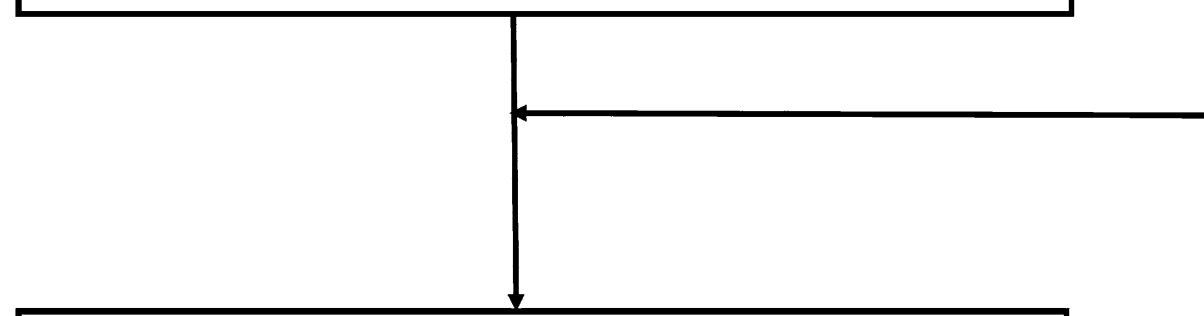

Identify the best and the worst solutions among all candidate solutions

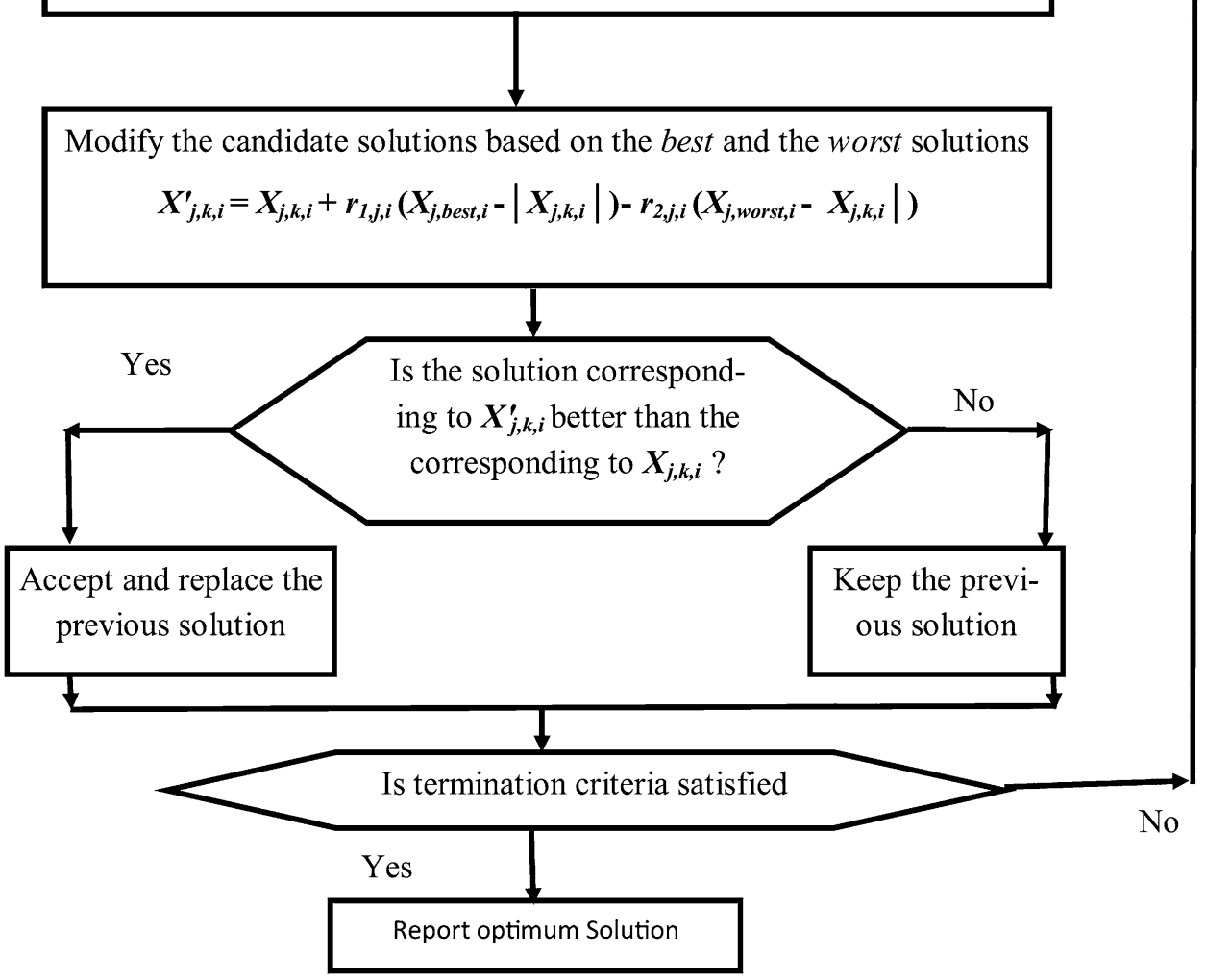

Now Consider two random numbers $r 1=0.7332, r 2=$ 0.4231 . Calculate the new value of all parameters candidate as per equation (7). All values of newly calculated parameters should be within the range as per Table 1. If new values fall below the lower limit then assign a lower limit to a new variables value. If new values of variable go beyond the upper limit of variables then assign an upper limit to the given variable as per Table 1.

$$
\begin{aligned}
X^{\prime} j, k, i & =X j, k, i+r 1, j, i(X j, \text { best }, i-|X j, k, i|) \\
& -r 2, j, i(X j, \text { worst }, i-|X j, k, i|)
\end{aligned}
$$

Candidate $1 \mathrm{I} p=7.7104+0.7332(4.9262-|7.7104|)$

$-0.4231(4.8570-|7.7104|)=6.8764$

\section{SN Applied Sciences}

Candidate $2 \mathrm{Ip}=4.1532+0.7332(4.9262-|4.1532|)$ $-0.4231(4.8570-|4.1532|)=4.4222$

Candidate $3 \mathrm{Ip}=4.0926+0.7332(4.9262-|4.0926|)-$ $0.4231(4.8570-|4.0926|)=4.3804$

Candidate $4 \mathrm{Ip}=4.9262+0.7332(4.9262-|4.9262|)-$ $0.4231(4.8570-|4.9262|)=4.9555$

Candidate $5 \mathrm{Ip}=4.8570+0.7332(4.9262-|4.8570|)-$ $0.4231(4.8570-|4.8570|)=4.9077$

Candidate 1 Ton $=112.6811+0.7332(142.7235-\mid 112$. $6811 \mid)-0.4231(67.9034-|112.6811|)=153.6536326($ $=150$ because maximum Ton $=150$ )

Candidate 2 Ton $=85.3170+0.7332(142.7235-\mid 85.317$ $0 \mid)-0.4231(67.9034-|85.3170|)=134.77514$ 
Table 3 Initial solution

\begin{tabular}{llrlll}
\hline Can. & Ip & \multicolumn{1}{l}{ Ton } & $\mathrm{t}(\%)$ & $\mathrm{V}$ & \multicolumn{1}{l}{ RWR } \\
\hline 1 & 7.7104 & 112.6811 & 49.9706 & 45.6465 & 11.4634 \\
2 & 4.1532 & 85.3170 & 47.0505 & 97.7998 & 15.7032 \\
3 & 4.0926 & 101.5753 & 41.7021 & 62.5484 & 23.0844 \\
4 & 4.9262 & 142.7235 & 47.8632 & 71.1816 & 24.7406 \\
5 & 4.8570 & 67.9034 & 39.2846 & 51.5042 & 9.8303 \\
\hline
\end{tabular}

Table 4 New values of all parameter and corresponding objective function RWR (first iteration)

\begin{tabular}{llllll}
\hline Can. & Ip & \multicolumn{1}{l}{ Ton } & $\mathrm{t}(\%)$ & $\mathrm{V}$ & $\mathrm{RWR}$ \\
\hline 1 & 6.8764 & 150.0000 & 50.0000 & 61.8904 & 18.0981 \\
2 & 4.4222 & 134.7751 & 50.0000 & 97.8713 & 22.5136 \\
3 & 4.3804 & 45.9918 & 47.2422 & 73.5511 & 29.4930 \\
4 & 4.9555 & 150.0000 & 50.0000 & 79.5073 & 25.4826 \\
5 & 4.9077 & 122.7613 & 45.5744 & 65.9316 & 21.0654 \\
\hline
\end{tabular}

Candidate 3Ton $=101.5753+0.7332(142.7235-\mid 101$. $5753 \mid)-0.4231(67.9034-|101.5753|)=103.6817411$

Candidate 4 Ton $=142.7235+0.7332(142.7235-\mid 142$. $7235 \mid)-0.4231(67.9034-|142.7235|)=174.3798843($ $=150$ because maximum Ton $=150)$

Candidate 5 Ton $=67.9034+0.7332(142.7235-\mid 67.903$ $4 \mid)-0.4231(67.9034-|67.9034|)=122.7614973$

Candidate $1 \mathrm{t}=49.9706+0.7332(47.38632-|49.9706|)$

$-0.4231(39.2846-|49.9706|)=52.9467(=50$ because maximum $\mathrm{t}=50$ )

Candidate $2 \mathrm{t}=47.0505+0.7332(47.38632-|47.0505|)$

$-0.4231(39.2846-|47.0505|)=50.9321(=50$ because maximum $\mathrm{t}=50$ )

Candidate $3 \mathrm{t}=41.7021+0.7332(47.38632-|41.7021|)$

$-0.4231(39.2846-|41.7021|)=47.2422$

Candidate $4 \mathrm{t}=47.8632+0.7332(47.38632-|47.8632|)$

$-0.4231(39.2846-|47.8632|)=51.4928(=50$ because maximum $\mathrm{t}=50$ )

Candidate $5 \mathrm{t}=39.2846+0.7332(47.38632-|39.2846|)$

$-0.4231(39.2846-|39.2846|)=45.5744$

Candidate $1 \mathrm{~V}=45.6465+0.7332(71.1816-|45.6465|)-$

$0.4231(51.5042-|45.6465|)=61.8904$

Candidate $2 \mathrm{~V}=97.7998+0.7332(71.1816-|97.7998|)-$

$0.4231(51.5042-|97.7998|)=97.8710$

Candidate $3 \mathrm{~V}=62.5484+0.7332(71.1816-|62.5484|)-$

$0.4231(51.5042-|62.5484|)=73.5510$

Candidate 4V $=71.1816+0.7332(71.1816-|71.1816|)-$ $0.4231(51.5042-|71.1816|)=79.5071$

Candidate $5 \mathrm{~V}=51.5042+0.7332(71.1816-|51.5042|)-$ $0.4231(51.5042-|51.5042|)=65.9316$

Now insert calculated new value into Table 4.
Table 5 Updated values of all variables and corresponding RWR (first iteration)

\begin{tabular}{llllll}
\hline Can. & Ip & Ton & $\mathrm{t}(\%)$ & $\mathrm{V}$ & $\mathrm{RWR}$ \\
\hline 1 & 6.8764 & 150 & 50 & 61.8904 & 18.0981 \\
2 & 4.4222 & 134.7751 & 50 & 97.8713 & 22.5136 \\
3 & 4.3804 & 145.9918 & 47.2422 & 73.5511 & 29.493 \\
4 & 4.9555 & 150 & 50 & 79.5073 & 25.4826 \\
5 & 4.9077 & 122.7613 & 45.5744 & 65.9316 & 21.0654 \\
\hline
\end{tabular}

Table 6 New values of all variables for second iteration

\begin{tabular}{llllll}
\hline Can. & Ip & Ton & $\mathrm{t}(\%)$ & $\mathrm{V}$ & $\mathrm{RWR}$ \\
\hline 1 & 5.6138 & 147.9726 & 48.605 & 67.7887 & 21.5808 \\
2 & 4 & 133.1297 & 48.605 & 100 & 24.9066 \\
3 & 4 & 144.065 & 45.9165 & 79.1568 & 31.7298 \\
4 & 4 & 147.9726 & 48.605 & 84.9635 & 32.1518 \\
5 & 4 & 121.4175 & 44.2905 & 71.7286 & 28.0169 \\
\hline
\end{tabular}

Table 7 Updated values of all variables and corresponding RWR for second iteration

\begin{tabular}{llllll}
\hline Can. & Ip & Ton & $\mathrm{t}(\%)$ & $\mathrm{V}$ & $\mathrm{RWR}$ \\
\hline 1 & 5.6138 & 147.9726 & 48.605 & 67.7887 & 21.5808 \\
2 & 4 & 133.1297 & 48.605 & 100 & 24.9066 \\
3 & 4 & 144.065 & 45.9165 & 79.1568 & 31.7298 \\
4 & 4 & 147.9726 & 48.605 & 84.9635 & 32.1518 \\
5 & 4 & 121.4175 & 44.2905 & 71.7286 & 28.0169 \\
\hline
\end{tabular}

Step 3: Compare every candidate and select with better RWR (objective function). Candidate 1 from Table 4 has better objective value compare to candidate 1 of Table 3 , hence inserted into Table 4 as candidate 1 . Similarly candidate $2,3,4$, and 5 of Table 4 has also better RWR as compare to candidate $2,3,4,5$ of Table 3 , hence candidate 2 , 3,4 and 5 of Table 3 inserted into Table 5 . First iteration is over.

Step 4: Consider Table 5 as input to second iteration. Consider $r 1=0.5058, r 2=0.4807$ and repeat the same procedure as per step 2 and step 3. Table 6 represent new value of all variables. Now compare each candidate from Table 5 and Table 6 . Select better RWR for each candidate and insert into Table 7.

\subsection{Optimization using TLBO algorithm}

Teaching-learning-based optimization (TLBO) is twophase optimization algorithm, first is teacher phase and second is the learner phase. Figure 3 shows flow diagram of TLBO Algorithm. 
Fig. 3 Flowchart of TLBO algorithm

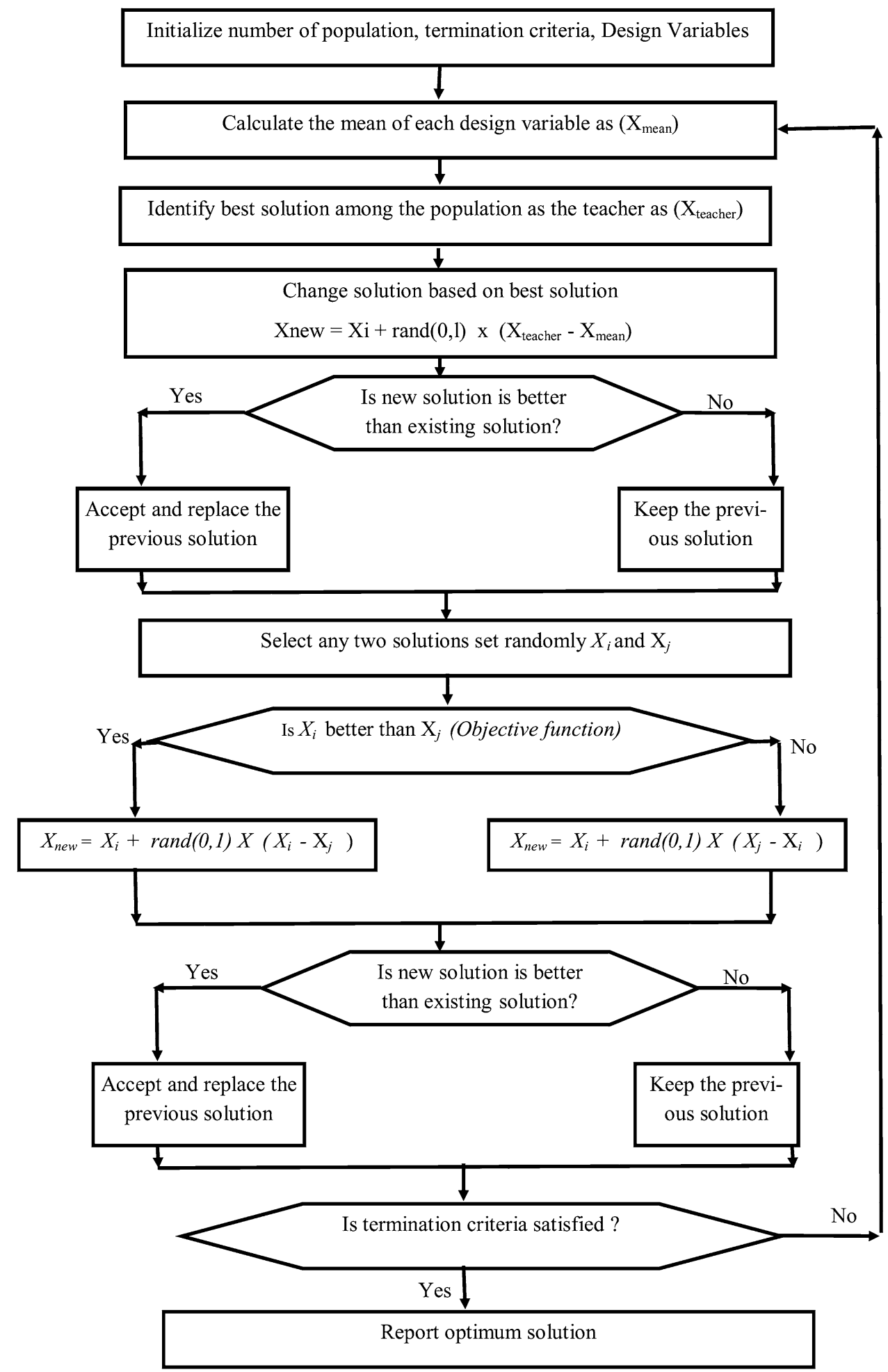

Step 1: Initial population is randomly generated in between upper bound and lower bound of variables as shown in Table 1. Calculate the mean of all variables.

Step 2: Objective is to maximize RWR as per equation 6. Candidate 2 has maximum RWR value hence selected as teacher. Value of Ip $=4.8153$, Ton $=85.5584, t=33.0294$,
$\mathrm{V}=46.5677$ selected as teacher's variables value. Calculated mean value of Ip, Ton, $t$ and $V$ are 6.2326, 87.24592, $34.22178,67.04024$ respectively. Consider four random numbers $r 1=0.6476, r 2=0.9520 \mathrm{r} 3=0.1991$ and $r 4=$ 0.0000 for $\mathrm{lp}$, Ton, $\mathrm{t}$ and $\mathrm{V}$ respectively. The difference mean values of $\mathrm{Ip}$, Ton, $\mathrm{t}$ and $\mathrm{V}$ calculated as following. 
Fig. 4 Effect of various control parameters on RWR

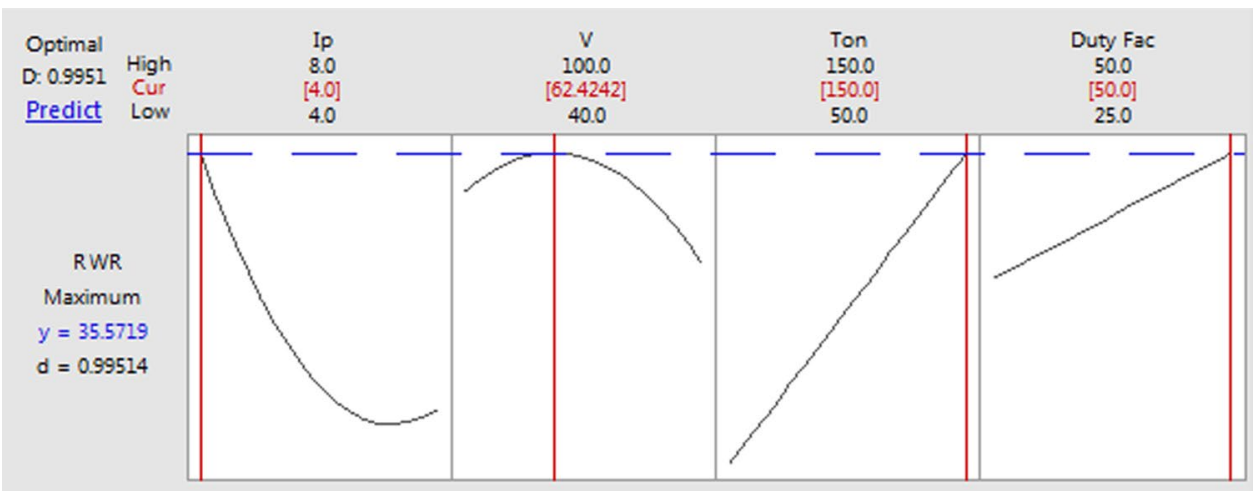

Table 8 Initial population

\begin{tabular}{llcllr}
\hline Can. & Ip & Ton & t (\%) & V & \multicolumn{1}{l}{ RWR } \\
\hline 1 & 5.8033 & 75.6442 & 30.6602 & 40.7974 & 2.7112 \\
2 & 4.8153 & 85.5584 & 33.0294 & 46.5677 & 10.8899 \\
3 & 6.1957 & 76.3754 & 30.6019 & 83.3591 & 5.7567 \\
4 & 7.6135 & 135.5253 & 30.5221 & 99.1143 & 6.7809 \\
5 & 6.7352 & 63.1263 & 46.2953 & 65.3627 & 6.9415 \\
Mean & 6.2326 & 87.24592 & 34.22178 & 67.04024 & 6.2326 \\
\hline
\end{tabular}

Table 9 New values of the input variables and the objective function (teacher phase)

\begin{tabular}{llrllr}
\hline Can. & Ip & Ton & $\mathrm{t}(\%)$ & $\mathrm{V}$ & \multicolumn{1}{c}{ RWR } \\
\hline 1 & 4.8854 & 74.0377 & 30.4228 & 40 & 6.5787 \\
2 & 4 & 83.9519 & 32.792 & 40 & 15.5988 \\
3 & 5.2778 & 74.7689 & 30.3645 & 75.9497 & 8.7602 \\
4 & 6.6956 & 133.9188 & 30.2848 & 91.7049 & 7.9195 \\
5 & 5.8174 & 61.5198 & 46.058 & 57.9533 & 6.6744 \\
\hline
\end{tabular}

Difference mean $(\mathrm{Ip})=0.6476 *(4.8153-6.2326)$

Difference mean $($ Ton $)=0.9520^{*}(85.5584-87.24592)$

Difference mean $(t)=0.1991 *(33.0294-34.22178)$

Difference mean $(\mathrm{V})=0.0000 *(46.5677-67.04024)$

The values of difference mean (Ip) is added to all parameter of Ip (first column) similarly difference mean (Ton) is added to all values of Ton (second column), difference mean $(t)$ is added to all values of $t$ and difference mean (V) is added to all values of $\mathrm{V}$ of Table 8 . Thus Table 9 is prepared shows the new values of all variables.

Step 3: Now compare Tables 8 and 9 for better values of RWR. The better value of RWR is placed in Table 10 for each candidate. Table 9 has a better RWR value for each candidate as compared to Table 8 . Hence select all
Table 10 Updated values of the variables and the objective function based on best of two (teacher phase)

\begin{tabular}{llrllr}
\hline Can. & Ip & Ton & t (\%) & V & \multicolumn{1}{l}{ RWR } \\
\hline 1 & 4.8854 & 74.0377 & 30.4228 & 40 & 6.5787 \\
2 & 4 & 83.9519 & 32.792 & 40 & 15.5988 \\
3 & 5.2778 & 74.7689 & 30.3645 & 75.9497 & 8.7602 \\
4 & 6.6956 & 133.9188 & 30.2848 & 91.7049 & 7.9195 \\
5 & 6.7352 & 63.1263 & 46.2953 & 65.3627 & 6.9415 \\
\hline
\end{tabular}

candidate from Table 9 and inserted into Table 10. The teacher phase is over now.

Step 4: Learner phase is started now. Every learner (candidate) has to interact with any other learner (candidate) and every learner interacts with anyone learner. Here, the interaction between learner 1 and learner 2, learner 2 and learner 3, learner 3 and Lerner 4, learner 4 and learner 5 and interaction between learner 5 and learner 1 is considered. Consider random numbers $\mathrm{r} 5=$ $0.3653, \mathrm{r} 6=0.1889, \mathrm{r} 7=0.2440$ and $\mathrm{r} 8=0.5060$ for $\mathrm{lp}$, Ton, $t$ and $V$ respectively.

After a comparison between learner 1 and learner 2, learner 2 has better RWR hence

(Ip) new for learner $1=4.8854+r 5^{*}(4-4.8854)=4.5621$

(Ton) new for learner $1=74.0377+r 6^{*}(83.9519-74.0377)$

$=75.9102$

(t) new for learner $1=30.4228+r 7^{*}(32.792-30.4228)=$ 31.0009

(V) new for learner $1=40+r 8^{*}(40-40)=40$

Similarly calculated new values of all variables of candidate 2 , candidate 3 , candidate 4 , and candidate 5 is inserted into Table 11. It is noted that the new value of variables should be within the range of input as per Table 1. If the value falls below lower limit the assign lower bound and if 
Table 11 New values of the variables and the objective function (learner phase)

\begin{tabular}{llrllr}
\hline Can. & Ip & Ton & $\mathrm{t}(\%)$ & $\mathrm{V}$ & \multicolumn{1}{c}{ RWR } \\
\hline 1 & 4.5621 & 75.9102 & 31.0009 & 40 & 9.1741 \\
2 & 4 & 85.6863 & 33.3843 & 40 & 16.0114 \\
3 & 5.7955 & 85.9405 & 30.3451 & 83.9219 & 7.2491 \\
4 & 6.6811 & 147.2893 & 26.3784 & 100 & 5.0965 \\
5 & 7.4107 & 61.0655 & 50 & 78.1963 & 8.7936 \\
\hline
\end{tabular}

it goes beyond the upper limit then assign an upper limit to the variable.

Step 5: Now compare Tables 10 and 11 for better values of $f(x)$ and better value of $f(x)$ is placed in Table 12 for each candidate. Learner phase is over now. Iteration one is over.

Step 6: Table 12 would be input to next iteration and repeat step 2,3,4 and step 5. Continue procedure for 100 iterations and report optimum solution.

\subsection{Optimization using Minitab 18}

Response optimizer of commercial software Minitab 18 optimizes response measures RWR $=35.5719$ and corresponding four control parameters are $I p=4 \mathrm{~A}, \mathrm{~V}=62.4242$ $\mathrm{v}$, Ton $=150 \mu$ seconds and $\mathrm{t}=50 \%$.

\section{Discussion}

MRR increases with the increase of peak current (Ip), but TWR also increases with Ip increases [10]. Many authors maximize MRR with compromisation to TWR and quality. The quality of the machined surface deteriorates if TWR has a higher value. Hence in this study, we consider RWR $(R W R=M R R / T W R)$ optimization. This optimization ensures to maximize MRR while the quality of the machined surface maintained at the best level. This section discusses the effect of four control parameters individually on RWR. Figure 4 shows the effect of all parameters on RWR value. RWR decreases with the increment of peak current (Ip),

Table 12 Updated values of the variables and the objective function based on fitness comparison (learner phase)

\begin{tabular}{llrllr}
\hline Can. & Ip & Ton & t (\%) & V & \multicolumn{1}{l}{ RWR } \\
\hline 1 & 4.5621 & 75.9102 & 31.0009 & 40 & 9.1741 \\
2 & 4 & 85.6863 & 33.3843 & 40 & 16.0114 \\
3 & 5.2778 & 74.7689 & 30.3645 & 75.9497 & 8.7602 \\
4 & 6.6956 & 133.9188 & 30.2848 & 91.7049 & 7.9195 \\
5 & 7.4107 & 61.0655 & 50 & 78.1963 & 8.7936 \\
\hline
\end{tabular}

Table 13 Optimum result

\begin{tabular}{llllll}
\hline Algorithm & $\mathrm{lp}$ & Ton & $\mathrm{t}(\%)$ & $\mathrm{V}$ & $\mathrm{RWR}$ \\
\hline Jaya Algorithm & 4 & 150 & 50 & 62.4614 & 35.6021 \\
TLBO & 4 & 150 & 50 & 62.4564 & 35.6021 \\
Response Opt. & 4 & 150 & 50 & 62.4242 & 35.5719 \\
\hline
\end{tabular}

RWR increases with the increases of pulse on time (Ton), RWR increases with the increases of duty factor $(t)$ and the optimum value of voltage $(\mathrm{V})$ observes at $\mathrm{V}=62.4242 \mathrm{~V}$, as shown in Figure 4.

\section{Results}

Optimization results are shown in tabular form. Table 13 shows the comparison and result produced by the different optimization algorithm.

\section{Conclusion}

The optimization of RWR using response surface modeling, Jaya Algorithm and teaching-learning-based optimization algorithm (TLBO) has been done. All three optimization methods show the same result. Maximum RWR is 35.6021 at an optimum level of $\mathrm{Ip}=4 \mathrm{amp}$, Ton $=150 \mu \mathrm{s}$, duty factor $\mathrm{t}=50 \%$ and $\mathrm{V}=62.4614 \mathrm{~V}$.

Jaya Algorithm converges the optimization quickly in just 15 iterations and found suitable for engineering optimization. RWR decreases with the increase of peak current, RWR increases with the increases of Ton and duty factor (t) while the optimum value of voltage (V) observed at $62.4614 \mathrm{~V}$.

Acknowledgements The authors would like to thanks the anonymous reviewers for their constructive comments.

\section{Compliance with ethical standards}

Conflict of interest The authors declare that they have no conflict of interest.

\section{References}

1. Jain Vijay Kumar (2009) Advanced machining processes. Allied Publishers, New Delhi

2. Davim JP (2013) Nontraditional machining processes. In: Manufacturing process selection handbook, pp 205-226

3. Ho KH, Newman ST (2003) State of the art electrical discharge machining (EDM). Int J Mach Tools Manuf 43(13):1287-1300 
4. Ho KH et al (2004) State of the art in wire electrical discharge machining (WEDM). Int J Mach Tools Manuf 44(12-13):1247-1259

5. Shabgard MR, Gholipoor A, Baseri H (2016) A review on recent developments in machining methods based on electrical discharge phenomena. Int J Adv Manuf Technol 87(5-8):2081-2097

6. Abbas NM, Solomon DG, Bahari MdF (2007) A review on current research trends in electrical discharge machining (EDM). Int J Mach Tools Manuf 47:1214-1228

7. Teimouri R, Baseri H (2012) Study of tool wear and overcut in EDM process with rotary tool and magnetic field. Adv Tribol 2012:1-8

8. Lee SH, Li XP (2001) Study of the effect of machining parameters on the machining characteristics in electrical discharge machining of tungsten carbide. J Mater Process Technol 115(3):344-358

9. Qudeiri JEA, Mourad A-HI, Ziout A, Abidi MH, Elkaseer A (2018) Electric discharge machining of titanium and its alloys: review. Int J Adv Manuf Technol 96:1319-1339

10. Muthuramalingam T, Mohan B (2015) A review on influence of electrical process parameters in EDM process. Arch Civ Mech Eng 15(1):87-94

11. Ghoreishi M, Atkinson J (2002) A comparative experimental study of machining characteristics in vibratory, rotary and vibro-rotary electro-discharge machining. J Mater Process Tech 120:374-384

12. Pradhan MK, Biswas CK (2011) Multi-response optimisation of EDM of AISI D2 tool steel using response surface methodology. Int J Mach Mach Mater 9(1-2):66-85

13. Kumar S, Singh R, Batish A, Singh TP (2012) Electric discharge machining of titanium and its alloys: a review. Int J Mach Mach Mater 11:84-111
14. Montgomery DC (1997) Design and analysis of experiments. Wiley, New York

15. Rajesh R, Anand MD (2012) The optimization of the electro-discharge machining process using response surface methodology and genetic algorithms. Proc Eng 38:3941-3950

16. Kao JY et al (2010) Optimization of the EDM parameters on machining Ti-6Al-4V with multiple quality characteristics. Int J Adv Manuf Technol 47(1-4):395-402

17. Tarng YS, Ma SC, Chung LK (1995) Determination of optimal cutting parameters in wire electrical discharge machining. Int J Mach Tools Manuf 35(12):1693-1701

18. Rao RV (2016) Jaya: A simple and new optimization algorithm for solving constrained and unconstrained optimization problems. Int J Ind Eng Comput 7:19-34

19. Rao RV (2019) Jaya: an advanced optimization algorithm and its engineering applications. Springer, Cham

20. Rao RV, Taler J, (eds) (2020) Advanced engineering optimization through intelligent techniques: select proceedings of AEOTIT 2018, vol 949. Springer, Berlin

21. Rao RV, Savsani VJ, Vakharia DP (2011) Teaching learning-based optimization: a novel method for constrained mechanical design optimization problems. Comput-Aided Des 43:303-315

22. Rao RV (2016) Teaching-learning-based optimization algorithm. Teaching learning based optimization algorithm. Springer, Cham, pp 9-39

Publisher's Note Springer Nature remains neutral with regard to jurisdictional claims in published maps and institutional affiliations. 